\title{
Domain-specific cognitive recovery after first-ever stroke: A follow-up study of 111 cases
}

\author{
G.M.S. NYS,,$^{1,2}$ M.J.E. VAN ZANDVOORT, ${ }^{1,2}$ P.L.M. DE KORT, ${ }^{3,4}$ B.P.W. JANSEN, ${ }^{4}$ \\ H.B. VAN DER WORP, ${ }^{2}$ L.J. KAPPELLE, ${ }^{2}$ AND E.H.F. DE HAAN, ${ }^{1,2}$ \\ ${ }^{1}$ Psychological Laboratory, Helmholtz Institute, Utrecht University, The Netherlands \\ ${ }^{2}$ Department of Neurology, University Medical Centre Utrecht, The Netherlands \\ ${ }^{3}$ Department of Neurology, St. Elisabeth Hospital, Tilburg, The Netherlands \\ ${ }^{4}$ Department of Neurology, Tweesteden Hospital, Tilburg, The Netherlands
}

(Received March 9, 2005; Final Revision August 10, 2005; Accepted August 11, 2005)

\begin{abstract}
The objective of this study is to examine the prognosis of acute cognitive disorders post-stroke, and to evaluate which clinical factors predict domain-specific cognitive recovery. We followed the course of cognitive functioning in 111 stroke patients and 77 healthy controls by administering two neuropsychological examinations with a 6 to 10 month interval (mean interval, $7.5 \pm 1.3$ months). The baseline examination was administered within three weeks post-stroke (mean interval, $7.9 \pm 4.2$ days). To examine determinants of domain-specific cognitive recovery, we recorded vascular risk factors, clinical variables, and lesion characteristics. Recovery in visual perception/construction (83\%) and visual memory $(78 \%)$ was the most common. An acute cognitive disorder predicted a long-term disorder in the same domain (all $p<.05$ ), except for visual perception/construction. Factors associated with poor cognitive recovery were age (all $p<.01$ ), preexistent verbal ability (all $p<.005$ ), lesion locations involving the temporal (all $p<.05$ ), frontal $(p<.05)$ and occipital lobe (all $p<.05)$, lesion volume $(p \leq .001)$, and diabetes mellitus $(p<.01)$. An early neuropsychological examination provides valuable information on long-term cognitive performance. The prognosis of higher-level visual disorders is the most favorable. Cognitive recovery is associated with age, preexistent ability, lesion volume, lesion location, and diabetes mellitus. (JINS, 2005, 11, 795-806.)
\end{abstract}

Keywords: Cerebrovascular disease, Cognition, Longitudinal, Acute, Determinants, Prognosis

\section{INTRODUCTION}

Cognitive dysfunction is a common sequel of stroke and affects up to two-thirds of patients (Ballard et al., 2003). In the acute phase of stroke cognitive impairment is related to direct local effects of the stroke, but also to hypoperfusion (Hillis et al., 2003, 2004) and functional deactivation (diaschisis) in nearby or remote areas of the brain (Ferro, 2001). Large variability in cognitive function exists among acute stroke patients, depending in part on the location and size of the lesion. Moreover, the degree of cognitive recovery within the first months after stroke varies considerably across patients (Ballard et al., 2003; Rasquin et al., 2004). Early diagnosis and prediction of the potential for recovery from cognitive deficits such as amnesia or executive dys-

Reprint requests to: Dr. G.M.S. Nys, Psychological Laboratory (17.24B), Utrecht University, Heidelberglaan 2, 3584 CS Utrecht, The Netherlands. E-mail: g.nys@fss.uu.nl function could be of great importance for determining an appropriate discharge destination and for guiding rehabilitation therapy.

While previous studies have demonstrated that a cognitive deterioration within the first three months post-stroke is a good predictor of an adverse functional outcome (Galski et al., 1993; Tatemichi et al., 1994; Mok et al., 2004; Nys et al., 2005) and post-stroke dementia (Lin et al., 2003), it is not known whether a detailed evaluation of specific cognitive functions in the early phase of stroke can give valid information about domain-specific cognitive functioning in the long term. Moreover, the prevalence of cognitive recovery and the cognitive abilities most likely to recover have not been adequately investigated, and it is unclear which clinical factors influence cognitive recovery after stroke. To our knowledge, only three studies (Desmond et al., 1996; Hochstenbach et al., 2003; Patel et al., 2003) examined potential predictors of cognitive recovery. In these studies, an association was demonstrated with smoking (Patel 
et al., 2003), unilateral neglect (Patel et al., 2003), diabetes mellitus (Desmond et al., 1996), lowered consciousness at hospital admission (Hochstenbach et al., 2003), and lesion side, with evidence of either a left hemisphere (Desmond et al., 1996) or a right hemisphere advantage (Hochstenbach et al., 2003; Patel et al., 2003). However, these studies suffered from a number of methodological shortcomings. First, baseline examination was typically performed at three months post-stroke or even at a later stage. Given that the largest improvement in cognitive functioning occurs within the first three months after stroke (Laska et al., 2001; Pedersen et al., 1995), the prevalence and degree of cognitive recovery may have been underestimated and several factors possibly associated with recovery may have been disregarded. Second, most longitudinal stroke studies (Hochstenbach et al., 2003; Patel et al., 2003) did not include a reexamination of the control group although this is crucial to control for potential practice effects and statistical artefacts. Third, none of the aforementioned studies have examined preexistent cognitive functioning of stroke patients. Therefore, these studies may have included patients with preexistent dementia or cognitive decline not related to the stroke, which has been shown to be present in about one sixth of stroke patients (Henon et al., 1997). Finally, these studies typically evaluated the prevalence of cognitive recovery in a cohort of stroke patients that included patients without cognitive disturbances (Desmond et al., 1996; Hochstenbach et al., 2003; Patel et al., 2003). Yet, such patients already perform very well at the baseline assessment and are therefore not likely to show improvement at the follow-up examination. Alternatively however, initially intact patients may show a subtle insidious decline in cognitive performance as compared to healthy controls. Therefore, it might be important to follow the cognitive recovery course separately in patients with cognitive impairment at baseline and those without impairment.

The aim of the present study was threefold: (1) to evaluate the prevalence and nature of cognitive recovery during the first months after stroke, (2) to examine the predictive value of a neuropsychological examination in the early phase of stroke with respect to domain-specific cognitive functioning after six months, and (3) to find factors associated with domain-specific cognitive recovery.

In the present study, the baseline neuropsychological assessment was performed within the first three weeks poststroke. We followed the course of six distinct cognitive domains in three participant groups, that is, healthy controls, patients who were cognitively intact at baseline, and patients who were cognitively impaired at baseline.

\section{METHODS}

\section{Research Participants}

We assembled our patient sample by applying predefined in- and exclusion criteria to a series of consecutive firstever stroke patients admitted to stroke units of three hospi- tals in the Netherlands (St. Elisabeth Hospital Tilburg, University Medical Centre Utrecht, and Tweesteden Hospital Tilburg) between December 2001 and October 2003. The diagnosis of stroke was based on the presence of both an acute focal deficit and an associated lesion on computed tomography (CT) or magnetic resonance imaging (MRI) scans. Patients with a normal scan at admission underwent a second scan within the first week post-stroke. Patients with preexistent neurological or psychiatric illnesses (as described in the patient's medical file) were excluded. In addition, patients with clinically manifest changes before the stroke and evidence on CT/MRI scans of silent infarcts, white matter lesions, transient ischemic attacks (TIAs), or cerebral atrophy were excluded from this study so that preexistent pathology could be considered silent. To this end, patients were interviewed on pre-stroke changes in cognitive, functional, or emotional status. In addition, patients with evidence of preexistent dementia (as defined by a score of 3.6 or higher on the short Informant Questionnaire on Cognitive Decline in the Elderly-IQCODE Dutch Version) (de Jonghe et al., 1997) were excluded. This questionnaire, administered to a close relative, consists of 16 questions concerning the changes experienced by the patient over the last 10 years before the stroke in aspects of daily behavior requiring memory and other intellectual abilities. Only native Dutch speakers were included, and all included participants were younger than 85 years to avoid disproportional aging effects on cognitive performance. Altogether, this procedure resulted in a population of 190 patients with acute first-ever stroke. In addition, we excluded patients who could not be neuropsychologically assessed within the first 21 days post-stroke on the majority of neuropsychological tasks to allow evaluation on at least 4 of 6 cognitive domains $(n=22)$. Further exclusions between baseline and follow-up examination because of events that could potentially affect cognitive recovery (recurrent stroke, psychosis, or interventions for severe comorbidity such as chemotherapy or coronary artery bypass grafting $(\mathrm{CABG}))(n=26)$ and other dropout reasons (death, refusal, detention in prison, or moving abroad) $(n=31)$ resulted in a study population of 111 patients. This population has been reported in a previous publication from our group (Nys et al., 2005).

The control group consisted of participants living in the community. The controls were either spouses or family of patients, or volunteers who came to our attention through advertising in newspapers or by word of mouth. First, a checklist was administered on the phone to exclude people with a neurological or psychiatric history, or with cognitive decline in the past ten years. This exclusion procedure resulted in a study population of 77 healthy controls. Control participants were comparable to the stroke patients with respect to age, sex, and education.

The ethics committees of the three participating hospitals approved the study protocol. Informed consent was obtained from all participants before inclusion in the study. 


\section{Longitudinal Neuropsychological Examination}

All participants underwent two subsequent neuropsychological examinations. The baseline neuropsychological examination of the stroke patients was carried out within the first three weeks after the stroke $(7.9 \pm 4.2$ days since stroke onset). All participants, including controls, were reexamined with an extended neuropsychological examination after a minimum of 6 and a maximum of 10 months, dated from their original assessment (mean interval, $7.5 \pm$ 1.3 months). The test battery (Lezak et al., 2004) was extended at the second examination where possible to have a more detailed and complete representation of each domain, so as to create a "gold standard" assessment of cognitive functioning (Table 1). The first assessment took about 1.5 hours, whereas the second extended examination lasted approximately 2.5 hours. Trained neuropsychologists performed the assessments. Participants were given breaks where appropriate to minimize the effects of fatigue or motivation on performance. Six major cognitive domains were assessed using both verbal and nonverbal neuropsychological tasks, that is, abstract reasoning, verbal short- and longterm memory, visual short- and long-term memory, language, executive functioning, and visual perception and construction (Table 1). We split memory into a verbal and a nonverbal domain to obtain at least one valid measure of memory for aphasic patients and neglect patients.

\section{Transformation of Neuropsychological Test Results}

Two types of variables were calculated from these test results: (1) Summary scores for the six cognitive domains were constructed for use in analyses to enhance reliability and to reduce the number of variables (Lezak et al., 2004). Each domain score was created by converting the raw scores from the individual tests to standardised scores ( $z$-scores) based on the means and standard deviations of the control group on the first and second examination. Subsequently, we averaged $z$-scores of tasks belonging to the same cognitive domain, in which a lower domain score indicates worse performance. (2) The prevalence of domain-specific disorders at baseline and at follow-up was calculated. A disorder was considered to be present whenever a patient's domain score was lower than -1.65 (Lezak et al., 2004), which is associated with the .05 level of statistical significance (Clark-

Table 1. Longitudinal neuropsychological examination (Lezak et al., 2004)

\begin{tabular}{|c|c|c|}
\hline & $\begin{array}{c}\text { Baseline } \\
\text { (mean interval } 8 \text { days post-stroke) }\end{array}$ & $\begin{array}{c}\text { Follow-up } \\
\text { (mean interval } 7.5 \text { months post-stroke) }\end{array}$ \\
\hline Reasoning & $\begin{array}{l}\text { Raven Advanced Progressive Matrices } \\
\quad \text { (short form) } \\
\text { Similarities WAIS-III }\end{array}$ & $\begin{array}{l}\text { Raven Advanced Progressive Matrices } \\
\quad \text { (short form) } \\
\text { Similarities WAIS-III }\end{array}$ \\
\hline Language & $\begin{array}{l}\text { Boston Naming Task (short form) } \\
\text { Token Test (short form) }\end{array}$ & $\begin{array}{l}\text { Boston Naming Task (short form)* } \\
\text { Token Test (short form) } \\
\text { Chapman Reading Task }\end{array}$ \\
\hline Verbal memory & $\begin{array}{l}\text { Digit Span } \ddagger \\
\text { R-AVLT } \ddagger\end{array}$ & $\begin{array}{l}\text { Digit Span } \ddagger \\
\text { R-AVLT* } * \\
\text { WMS-R Logical Memory I and II } \ddagger\end{array}$ \\
\hline Visual memory & $\begin{array}{l}\text { Corsi Block Span } \\
\text { Rey-O Delay }\end{array}$ & $\begin{array}{l}\text { Corsi Block Span } \\
\text { WMS-R Visual Reproduction } \neq \\
\text { modified Location Learning Task } \ddagger\end{array}$ \\
\hline Executive function & $\begin{array}{l}\text { Brixton Spatial Anticipation Test } \\
\text { Visual Elevator (TEA) } \ddagger \\
\text { Letter Fluency ( } \mathrm{N} \text { and } \mathrm{A})\end{array}$ & $\begin{array}{l}\text { Brixton Spatial Anticipation Test } \\
\text { Visual Elevator (TEA) } \ddagger \\
\text { Letter Fluency (N and A) } \\
\text { Semantic Fluency (Animals) } \\
\text { Stroop Colour Word Test } \\
\text { Zoo Test (BADS) }\end{array}$ \\
\hline Perception/construction & $\begin{array}{l}\text { Benton Line Orientation (short form) } \\
\text { Face Recognition (short form) } \\
\text { Rey-O Copy }\end{array}$ & $\begin{array}{l}\text { Benton Line Orientation (short form)* } \\
\text { Face Recognition (short form) } \\
\text { Block Design WAIS-III }\end{array}$ \\
\hline
\end{tabular}

*Alternate forms used at follow-up in both patients and controls. Abbreviations: WMS-R $=$ Wechsler Memory Scale-Revised; R-AVLT $=$ Rey Auditory Verbal Learning Test; Rey-O = Rey-Osterreith Complex Figure; TEA = Test of Everyday Attention (Robertson et al., 1994); BADS = Behavioural Assessment of the Dysexecutive Syndrome. Brief explanation unfamiliar tasks: Brixton Spatial Anticipation Test assesses nonverbal concept formation and flexibility, Visual Elevator assesses verbal cognitive flexibility, the Zoo Test (BADS) assesses planning; the modified Location Learning Task (Kessels et al., 2004) assesses immediate and long-term object-location memory; the Chapman Reading Task assesses language comprehension and reading.

$\ddagger$ Tasks consisting of multiple components: Digit span (forward and backward), R-AVLT (immediate recall, delayed recall, recognition), WMS-R Logical Memory (Immediate and Delayed Recall), WMS-R Visual Reproduction (Immediate and Delayed Recall), modified Location Learning Task (Immediate and Delayed Recall), Visual Elevator (accuracy and reaction time). 
Carter, 1997). Patients were classified as "cognitively impaired" if they demonstrated a disorder in at least one cognitive domain, whereas patients without any disorder were classified as "cognitively unimpaired." Although the latter may still show a subtle cognitive decline due to the stroke, their domain-specific performance fell within the normal range (Lezak et al., 2004).

\section{Potential Correlates of Domain-specific Cognitive Recovery}

\section{Demographic characteristics and preexistent ability}

We recorded the age and gender of all participants. Level of education was scored using a Dutch classification system according to Verhage, ranging in ascending order from 1 (less than primary school) to 7 (university degree). Preexistent verbal ability was estimated by means of the National Adult Reading Test (NART-Dutch Version) (Schmand et al., 1991) at the follow-up examination. This measure is known to be relatively unaffected by neurological disorder (Crawford et al., 1988; Watt \& O'Carroll, 1999), as well as when verbal skills are affected by brain damage (Crawford et al., 1988; Schmand et al., 1991). Particularly in older participant samples, this is a better measure of preexistent ability than the level of education.

\section{Stroke lesion characteristics}

Lesion characteristics were determined from CT or MRI scans by an experienced stroke neurologist ( $\mathrm{HBvdW})$ who was blind for the clinical data. Stroke type (infarct, haemorrhage), lesion location (supratentorial, infratentorial), lesion side of supratentorial lesions (left, right), cortical or subcortical lobe involvement (frontal, parietal, temporal, occipital), subcortical gray matter involvement (caudate nucleus, striatum, thalamus), and infratentorial involvement (brain stem, cerebellum) were recorded. In addition, lesion volume was calculated with Leica Q500 MCP image analysis software by manual tracing of the lesion on each slice on which the infarct or intracerebral haemorrhage was present. This method is described in detail elsewhere and has been shown to have a high interrater reliability (van der Worp et al., 2001). Finally, the presence of preexistent brain pathology was recorded by using straightforward dichotomous ratings suitable for use with both CT and MRI. We rated (1) preexistent silent infarcts (classified as present when an infarct was found in the brain that could not have caused the actual neurological deficits and if the patient, family, or medical record did not describe a prior corresponding stroke episode) (Nys et al., 2005), (2) white matter lesions (scored as present if patients obtained a score $>0$ on the Van Swieten scale) (van Swieten et al., 1988), and (3) cortical atrophy (classified as present when a generalized dilatation of cortical sulci was found) (Nys et al., 2005).

\section{Vascular risk factors}

We recorded the presence of vascular risk factors on the basis of the medical history and medication use (history of diabetes mellitus, hypertension, hypercholesterolaemia, transient ischemic attack), and recorded whether patients had a history of smoking during the last five years and if they had an alcohol consumption of more than two units per day.

\section{Clinical status at baseline}

Stroke severity at baseline was assessed by means of the National Institutes of Health Stroke Scale (NIHSS) (Brott et al., 1989). The presence and severity of depressive symptoms at baseline was measured with the MontgomeryÅsberg Depression Rating Scale (MADRS) (Montgomery \& Åsberg, 1979). The MADRS is an observer-rated scale ranging from 0 (no depressive symptoms) to 60 (severe depressive symptoms), which is not heavily relying on somatic symptoms. The degree of unilateral inattention in the early phase of stroke was assessed by means of the Star Cancellation (Wilson et al., 1987), which is a cancellation task ranging from 0 (patient neglected all items) to 54 (patient cancelled out all items).

\section{Statistical analyses}

We evaluated differences among patients who were cognitively intact at baseline and those who were not by comparing clinical and demographic characteristics in both groups using $\chi^{2}$ tests for categorical data, Mann-Whitney $U$ Tests for ordinal data, and student $t$ tests for continuous data.

To examine Aim 1 (i.e., to evaluate the prevalence and nature of cognitive recovery during the 6-10 months after stroke), the longitudinal change in cognitive performance was calculated by comparing the difference in domain scores between the baseline and follow-up examination. Positive difference scores indicate improvement, whereas negative difference scores indicate decline from baseline. Scores near zero indicate a change that equals the mean control group change (which is zero as a result of our standardization method). A univariate analysis of variance (ANOVA) was performed to compare the extent of cognitive change in the six cognitive domains between controls, patients who were cognitively intact at baseline, and patients who were cognitively impaired at baseline.

To examine Aim 2 (i.e., to examine the predictive value of early cognitive testing with respect to cognitive functioning in the long term), logistic regression analyses were performed. A series of hierarchical logistic regression analyses were performed with age, gender, and estimated preexistent verbal ability (NART) entered in the first step and all acute cognitive deficits entered simultaneously in the next step in a forward stepwise fashion. Domain-specific cognitive performance at follow-up (dichotomous variable: impaired $v s$. unimpaired) was considered as the dependent (to be predicted) variable. 
To examine Aim 3 (i.e., to identify independent predictors of domain-specific cognitive change), we used hierarchical multiple linear regression analyses with the cognitive change scores as the dependent variables. Baseline cognitive performance and demographic variables were entered in the first step; candidate predictor variables were then entered separately in the next step. Although the use of adjustment for baseline cognitive performance is controversial, we believe such adjustment is necessary to take into account learning (the effect of familiarity with tests from the previous administration) and ceiling effects (those with a good baseline performance cannot substantially improve) and to reduce variability within participants. Finally, to identify independent predictors of domainspecific recovery, all variables with significant univariate associations $(p<.05)$ were entered together in a multivariate stepwise regression analysis for each cognitive domain.

\section{RESULTS}

\section{Cognitive Disorders in the Early Phase of Stroke}

Of the 168 stroke patients included in the early phase of stroke, 111 patients were reexamined at follow-up. In a prior publication on the same population (Nys et al., 2005), we examined the additional value of a neuropsychological examination in the prediction of cognitive impairment and activities of daily living (ADL) dependence for basic (e.g., personal hygiene, toilet use) and more complex activities (e.g., household management, social activities), beyond that of well-known demographic and medical predictors. Patients who were not reexamined in this cohort demonstrated significantly $(p<.05)$ more impairments in visual memory ( $32.7 \%$ vs. $16.8 \%)$, executive functioning (50.0\% vs. $30.3 \%)$, and visual perception and construction (49.1\% vs. $31.2 \%)$. Potential causes for this bias are that patients who were not reexamined were older $(67.9 \pm 12.5$ vs. $60.1 \pm 14.2$ ), and they demonstrated more silent infarct(s) $(27.5 \%$ vs. $13.7 \%)$ and white matter lesions (38.6\% vs. $15.3 \%)$. No association with characteristics directly related to the stroke lesion or to vascular risk factors could be demonstrated.

The clinical and demographic characteristics of the included patient population $(N=111)$ are shown in Table 2, classified according to unimpaired versus impaired cognitive functioning at baseline. Of the 111 patients, 54 (49\%) were cognitively impaired in one or more domains in the first weeks after stroke, with a mean of 3 impaired domains per patient [range: 1-6]. More specifically, 36 of 111 patients $(32.4 \%)$ demonstrated a disorder in visual perception and construction, 35 patients (31.5\%) in executive functioning, 27 patients $(24.3 \%)$ in abstract reasoning, 24 patients $(21.6 \%)$ in language, 24 patients (21.6\%) in verbal memory, and 18 patients $(16.2 \%)$ in visual memory (Figure 1). Patients with unimpaired cognition at baseline demonstrated a higher level of education and a smaller lesion volume than patients who were cognitively impaired in the early phase of stroke, and they less often demonstrated frontal, parietal, and temporal lesions. In addition, patients with unimpaired cognition at baseline were more often discharged home and received less therapy than cognitively impaired patients at baseline (Table 2).

\section{Prevalence of Cognitive Change between Baseline and Follow-up}

\section{Initially impaired patients}

Patients with a small number of cognitive impairments at baseline more often demonstrated a complete cognitive recovery in the long term than patients with a more widespread cognitive impairment (Mann-Whitney $U=172.5$; $p<.001)$. The mean number of cognitive disorders per patient decreased from $3.0[1-6]$ at baseline to $1.3[0-5]$ at follow-up $(p<.001)$. Domain-specific recovery in visual perception/construction and visual memory was the most frequent [i.e., 30 of the 36 patients (83\%) and 14 of the 18 patients $(78 \%)$, respectively], whereas recovery in abstract reasoning and language was the least common [i.e., 11 of the 27 patients (41\%) and 13 of the 24 patients (54\%), respectively]. At follow-up, 1 patient (3.7\%) had gained a disorder in abstract reasoning, 5 patients (16.7\%) in language, 1 patient $(3.3 \%)$ in verbal memory, 4 patients $(11.1 \%)$ in visual memory, and 1 patient (5.6\%) in visual perception and construction. There were no patients who gained a deficit in executive functioning.

\section{Initially unimpaired patients}

The majority of these patients $(51 / 57$ or $90 \%)$ remained cognitively unimpaired in the long term. At follow-up, 3 patients $(5.3 \%)$ had developed a deficit in abstract reasoning, 2 patients $(3.5 \%)$ in executive functioning, 1 patient $(1.8 \%)$ in visual memory, and 3 patients $(5.3 \%)$ in visual perception/construction. There were no initially unimpaired patients who gained a deficit in verbal memory or language.

For the two groups taken together, the neuropsychological profile at follow-up was different from the early phase of stroke, with disorders in abstract reasoning being the most common and disorders in visual and verbal memory being the least common (Figure 1).

When comparing the degree of cognitive change between controls, initially unimpaired, and initially impaired patients by means of an ANOVA, a main effect of group was found with respect to all cognitive domains (all $p<$ .01). Post-hoc Dunnett tests revealed that the initially impaired group demonstrated a significant improvement in all cognitive domains compared to healthy controls (all $p<.05$ ). In contrast, the initially unimpaired group showed the same longitudinal course as controls in all domains (Figure 2). 
Table 2. Patient characteristics at baseline

\begin{tabular}{|c|c|c|c|c|}
\hline Characteristics & $\begin{array}{l}\text { Entire population } \\
\qquad(N=111)\end{array}$ & $\begin{array}{l}\text { Unimpaired cognition } \\
\qquad(N=57)\end{array}$ & $\begin{array}{l}\text { Impaired cognition } \\
\qquad(N=54)\end{array}$ & $p$ \\
\hline \multicolumn{5}{|l|}{ Demographics } \\
\hline Age (years), mean $\pm S D$ & $60.1 \pm 14.2$ & $59.5 \pm 14.2$ & $60.8 \pm 14.2$ & 0.63 \\
\hline Education, median [range] & $4[0-7]$ & $5[2-7]$ & $4[1-7]$ & $0.001 *$ \\
\hline Gender, female & 45.9 & 40.4 & 51.9 & 0.22 \\
\hline \multicolumn{5}{|l|}{ Stroke lesion characteristics } \\
\hline$\%$ infarct/all strokes & 90.1 & 94.7 & 85.2 & 0.09 \\
\hline Lesion volume $(\mathrm{ml})$, mean $\pm S D$ & $17.8 \pm 29.2$ & $8.8 \pm 16.2$ & $27.4 \pm 36.3$ & $0.001 *$ \\
\hline$\%$ supratentorial/all strokes & 84.8 & 80.4 & 89.6 & 0.20 \\
\hline$\%$ left/left and right supratentorial & 51.7 & 53.3 & 50.0 & 0.74 \\
\hline \multicolumn{5}{|l|}{ Involvement of } \\
\hline Frontal lobe & 16.2 & 7 & 25.9 & $0.007 *$ \\
\hline Parietal lobe & 22.5 & 12.3 & 33.3 & $0.008 *$ \\
\hline Temporal lobe & 25.2 & 10.5 & 40.7 & $0.001 *$ \\
\hline Occipital lobe & 13.5 & 15.8 & 11.1 & 0.47 \\
\hline \multicolumn{5}{|l|}{ Preexistent pathology } \\
\hline White matter lesions & 15.3 & 15.8 & 14.8 & 0.89 \\
\hline Silent infarct(s) & 13.7 & 17.0 & 10.2 & 0.32 \\
\hline Cerebral atrophy & 10.7 & 11.3 & 10.0 & 0.83 \\
\hline \multicolumn{5}{|l|}{ Vascular risk factors } \\
\hline Diabetes mellitus & 10.9 & 12.3 & 9.4 & 0.63 \\
\hline Hypertension & 40.0 & 43.9 & 35.8 & 0.39 \\
\hline TIA(s) & 14.5 & 12.3 & 17.0 & 0.49 \\
\hline Hypercholesterolaemia & 17.3 & 21.1 & 13.2 & 0.28 \\
\hline Discharge destination & & & & $0.001 *$ \\
\hline Home & 58.2 & 57.4 & 39.6 & \\
\hline Rehabilitation center & 29.1 & 21.1 & 37.7 & \\
\hline Nursing home & 12.7 & 3.5 & 22.6 & \\
\hline \multicolumn{5}{|l|}{ Treatment } \\
\hline Physiotherapy & 73.6 & 66.1 & 81.5 & 0.07 \\
\hline Speech therapy & 40.0 & 26.8 & 53.7 & $0.004 *$ \\
\hline Occupational therapy & 50.9 & 39.3 & 63.0 & $0.01 *$ \\
\hline Cognitive therapy & 9.1 & 1.8 & 16.7 & $0.007 *$ \\
\hline
\end{tabular}

Values are within-group percentages unless indicated otherwise. Some percentages are based on incomplete samples due to small amounts of missing data. Analyses are chi square analyses for categorical data, Mann-Whitney $U$ Tests for ordinal data, and Student $t$ tests for continuous data between patients with intact and impaired cognition. $* p<.05$.

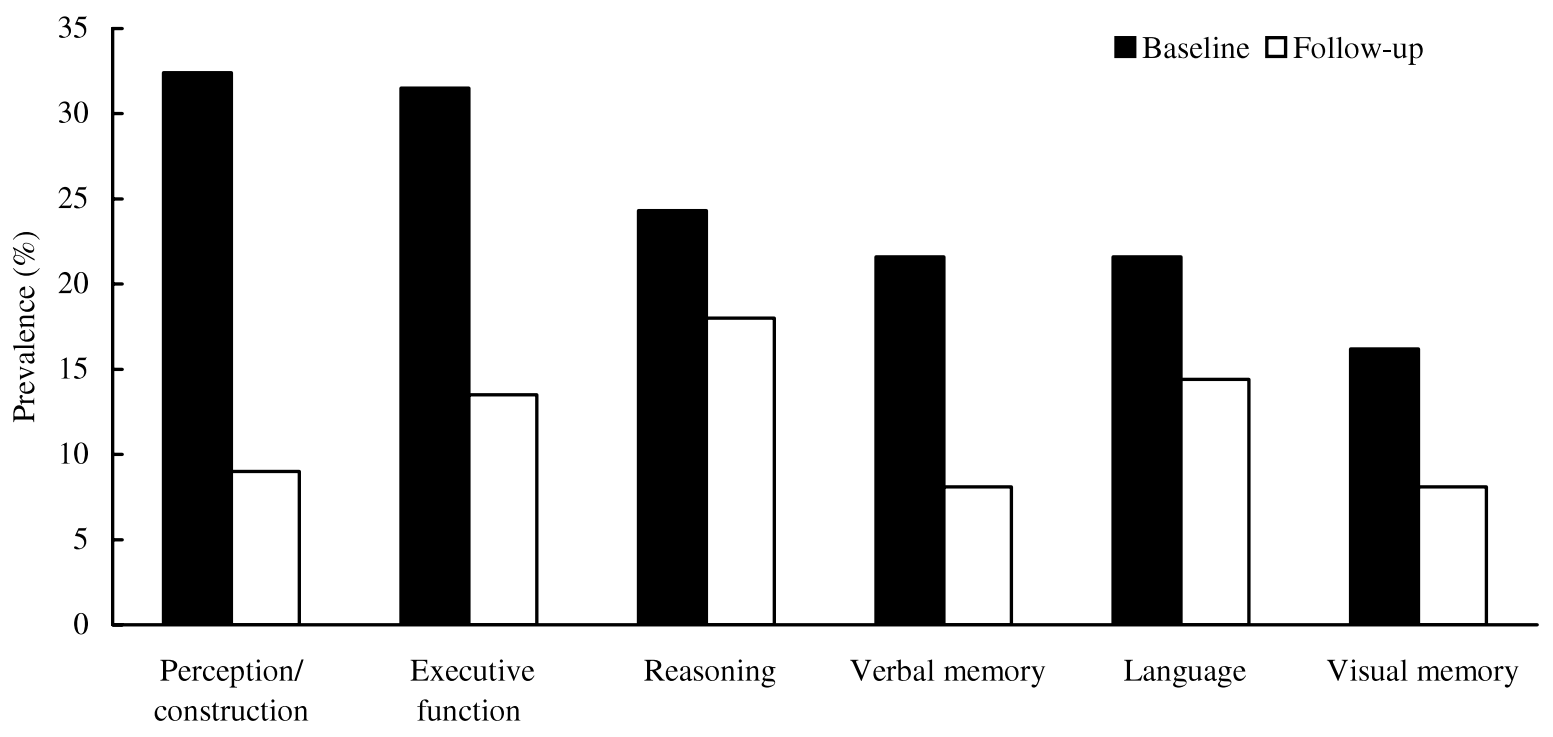

Fig. 1. Prevalence of cognitive disorders at baseline and follow-up $(N=111)$. 


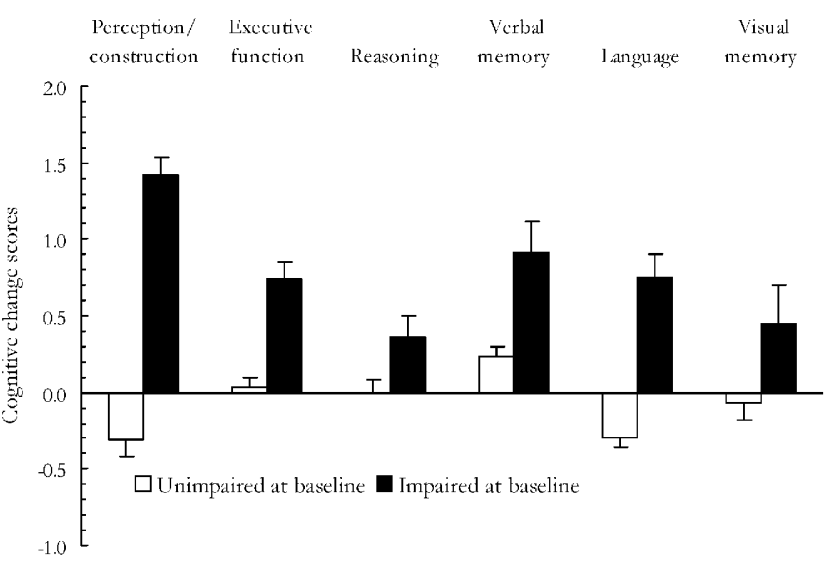

Fig. 2. Level of cognitive change between baseline and follow-up $(N=111)$.

\section{Predictive Value of the Neuropsychological Examination in the Early Phase of Stroke}

Multivariate stepwise analyses adjusted for age, gender, and NART score demonstrated that a domain-specific cognitive impairment at follow-up was best predicted by the same cognitive impairment at baseline, except for two domains. Language was better predicted by abstract reasoning at baseline, and visual perception/construction was better predicted by executive functioning at baseline (Table 3). Each model demonstrated a sufficient goodness-of-fit as measured with the Hosmer and Lemeshow goodness-of-fit test (all $p>$.05) (Hosmer \& Lemeshow, 1989). Although we cannot rule out the possibility that the NART is affected by aphasia and underestimates premorbid levels of functioning in that group, it should be noted that across cognitively impaired patients, NART scores were relatively unaffected by language impairment at baseline $[t(52)=0.52 ; p=.61]$ or at follow-up $[t(27)=0.65 ; p=.52]$. Therefore, we consider the NART to be a reasonable estimate of premorbid ability in patients with post-stroke language impairment.

\section{Clinical Correlates of Domain-specific Cognitive Recovery}

All demographic variables, lesion characteristics, and vascular risk factors that are related to recovery in one or more cognitive domains are shown in Table 4. Important demographic characteristics associated with poor cognitive recovery were lower NART score and older age. Lesion characteristics associated with poor recovery were a larger lesion volume and lesion locations involving the frontal, temporal, or occipital lobe. Diabetes mellitus was the only vascular risk factor associated with a poor recovery in a single cognitive domain (i.e., abstract reasoning). Finally, greater severity of unilateral neglect was related to a poor recovery in visual memory.

In the multivariate regression models (Table 5), a higher NART score was associated with recovery in 5 of the 6 cognitive domains. A younger age was associated with a

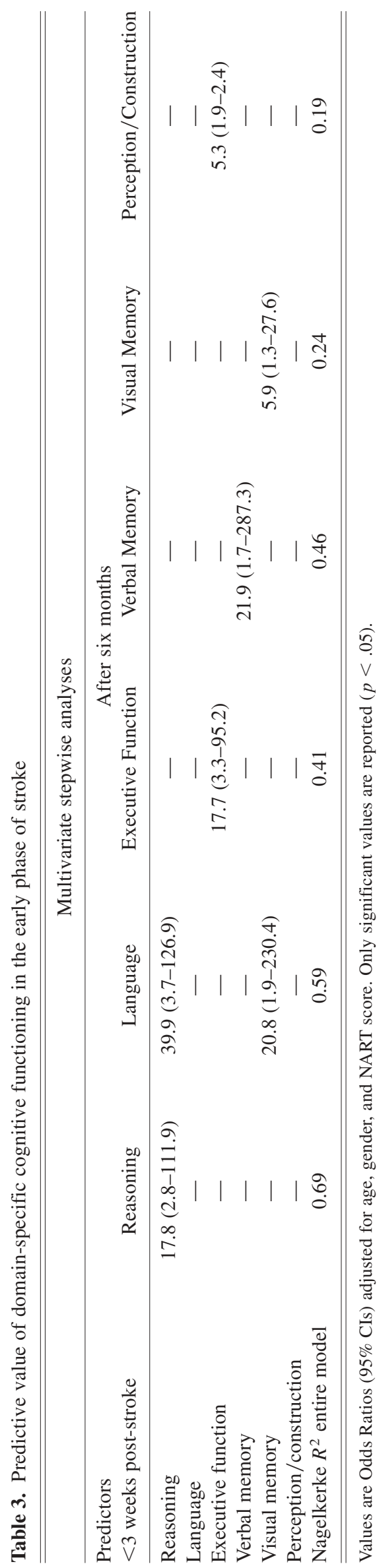


Table 4. Predictors of domain-specific cognitive change-univariate analyses for preselection of predictor variables

\begin{tabular}{|c|c|c|c|c|c|c|}
\hline & Reasoning & $\begin{array}{l}\text { Executive } \\
\text { Functioning }\end{array}$ & $\begin{array}{l}\text { Visual Perception/ } \\
\text { Construction }\end{array}$ & $\begin{array}{l}\text { Verbal } \\
\text { Memory }\end{array}$ & $\begin{array}{l}\text { Visual } \\
\text { Memory }\end{array}$ & Language \\
\hline \multicolumn{7}{|l|}{ Demographics $^{\dagger}$} \\
\hline Age, years & - & $-.25(.001)$ & - & $-.14(.039)$ & $-.23(.004)$ & $-.16(.02)$ \\
\hline Sex & - & - & - & - & - & - \\
\hline NART & $.46(.001)$ & $.26(.001)$ & - & $.29(.000)$ & $.24(.004)$ & $.38(.001)$ \\
\hline \multicolumn{7}{|l|}{ Vascular risk factors $\ddagger$} \\
\hline Hypertension & - & - & - & - & - & - \\
\hline Diabetes & $-.16(.04)$ & - & - & - & - & - \\
\hline Hypercholesterolaemia & - & - & - & - & - & - \\
\hline TIA $(\mathrm{s})$ & - & - & - & - & - & - \\
\hline Smoking & - & - & - & - & - & - \\
\hline \multirow{2}{*}{\multicolumn{7}{|c|}{ Lesion characteristics $\ddagger$}} \\
\hline & & & & & & \\
\hline Volume & - & $-.18(.02)$ & $-.19(.004)$ & - & $-.34(.001)$ & - \\
\hline Side & - & - & - & - & - & - \\
\hline Lesion type & - & - & - & - & - & - \\
\hline Supra-/Infratentorial & - & - & - & - & - & - \\
\hline \multicolumn{7}{|l|}{ Involvement of } \\
\hline Frontal lobe & $-.16(.04)$ & - & $-.20(.003)$ & - & - & - \\
\hline Parietal lobe & - & - & - & - & - & - \\
\hline Temporal lobe & - & $-.18(.02)$ & $-.13(.05)$ & $-.12(.014)$ & $-.19(.02)$ & - \\
\hline Occipital lobe & - & - & $-.16(.01)$ & - & $-.24(.002)$ & - \\
\hline \multicolumn{7}{|l|}{ Involvement of } \\
\hline Thalamus & - & - & - & - & - & - \\
\hline Caudate & - & - & - & - & - & - \\
\hline Striatum & - & - & - & - & - & - \\
\hline \multicolumn{7}{|l|}{ Involvement of } \\
\hline Brain stem & - & - & - & - & - & - \\
\hline Cerebellum & - & - & - & - & - & - \\
\hline Silent infarct(s) & - & - & - & - & - & - \\
\hline White matter lesions & - & - & - & - & - & - \\
\hline Cerebral atrophy & - & - & - & - & - & - \\
\hline Mood at baseline $\ddagger$ & - & - & - & - & - & - \\
\hline Unilateral attention & - & - & - & - & $.24(.005)$ & - \\
\hline Stroke severity $\ddagger$ & - & - & - & - & - & - \\
\hline
\end{tabular}

$\dagger$ Adjustment for baseline domain-specific cognitive functioning.

\$Adjustment for baseline domain-specific cognitive functioning, age, and NART score. Values are standardized beta’s ( $p$ values), and only values with $p<.05$ are reported.

good recovery in the two memory domains, language, and executive functioning. Lesion location was more important than lesion volume in predicting the degree of domainspecific recovery, that is, a lesion in the frontal lobe was independently associated with a poor recovery in visual perception and construction, a lesion in the temporal lobe with a poor recovery in executive function and verbal memory, and a lesion in the occipital lobe with a poor recovery in visual memory and visual perception/construction. A smaller lesion volume independently predicted a better recovery in visual memory. Finally, diabetes mellitus was independently related to a poor recovery in abstract reasoning.

\section{DISCUSSION}

The main reason why previous studies have paid little attention to cognition in the early phase of stroke has been uncertainty about the reliability and predictive value of such an early examination. It has been argued that a number of stroke-related problems such as fatigue, fluctuating level of arousal, or emotional distress may exacerbate or obscure cognitive performance in this stage (Lezak et al., 2004). Nevertheless, the present study demonstrates that an early concise neuropsychological examination can be used to predict long-term cognitive performance as assessed with a more extensive examination in patients with a first-ever stroke. Therefore, such an examination could assist in a more appropriate allocation of rehabilitation resources. Moreover, interventions aimed at restoration and/or compensation of impaired cognitive functions could start off in an earlier stage, which might be important for the effectiveness of the treatment (Paolucci et al., 2000; Biernaskie et al., 2004). There were two exceptions with respect to the specificity of the predictions, however. First, an executive dis- 
Table 5. Predictors of domain-specific cognitive change-multivariate analyses

\begin{tabular}{|c|c|c|c|c|c|c|}
\hline & Reasoning & $\begin{array}{c}\text { Executive } \\
\text { Functioning }\end{array}$ & $\begin{array}{l}\text { Visual Perception/ } \\
\text { Construction }\end{array}$ & $\begin{array}{l}\text { Verbal } \\
\text { Memory }\end{array}$ & $\begin{array}{l}\text { Visual } \\
\text { Memory }\end{array}$ & Language \\
\hline \multicolumn{7}{|l|}{ Demographics } \\
\hline Age, years & & $-.25(.001)$ & & $-.17(.001)$ & $-.21(.01)$ & $-.18(.001)$ \\
\hline NART & $.44(.001)$ & $.22(.004)$ & & $.26(.001)$ & $.21(.004)$ & $.35(.001)$ \\
\hline \multicolumn{7}{|l|}{ Vascular risk factors } \\
\hline Diabetes mellitus & $-.19(.01)$ & & & & & \\
\hline \multicolumn{7}{|l|}{ Lesion characteristics } \\
\hline Volume & & $\mathrm{X}$ & $\mathrm{X}$ & & $-.32(.001)$ & \\
\hline \multicolumn{7}{|l|}{ Involvement of } \\
\hline Frontal lobe & $\mathrm{X}$ & & $-.14(.02)$ & & & \\
\hline \multicolumn{7}{|l|}{ Parietal lobe } \\
\hline Temporal lobe & & $-.18(.02)$ & $\mathrm{X}$ & $-.12(.01)$ & $\mathrm{X}$ & \\
\hline Occipital lobe & & & $-.16(.01)$ & & $-.15(.05)$ & \\
\hline Unilateral attention & & & & & $\mathrm{X}$ & \\
\hline Adjusted $R^{2}$ entire model & .38 & .48 & .64 & .76 & .52 & .81 \\
\hline
\end{tabular}

Forward stepwise linear regression analyses with adjustment for baseline domain-specific cognitive functioning. Values are standardised beta's ( $p$-values), and $\mathrm{X}$ indicates predictors that do not reach significance in the multivariate model. NART denotes National Adult Reading Test.

order rather than perceptual performance in the early phase of stroke predicted the presence of a long-term perceptual/ constructional deficit. While there is some evidence that executive dysfunction is related to the severity of unilateral neglect after stroke (Manly et al., 2002; Rusconi et al., 2002), the present study shows that this also applies to visual perception and construction in general. Particularly, the constructional component in this domain may benefit from an intact planning capacity and the ability to use structure (Elderkin-Thompson et al., 2004). Second, abstract reasoning emerged as the best predictor of long-term language performance. In a similar vein, previous studies have shown a close association between abstract reasoning impairment and aphasia (De Renzi et al., 1966; Gainotti et al., 1986; Baldo et al., 2005).

The majority of patients who were cognitively unimpaired immediately after the stroke retained the same level of cognitive performance at follow-up as healthy controls, suggesting that there is no evidence of a generalized insidious cognitive deterioration in this stroke population, at least not in the first six months after the stroke. An important note here is that cognitive impairment was defined according to very stringent criteria (at least one domain score $<-1.65$ which is associated with the fifth percentile level of control performance). Therefore, some of these patients may have shown a more subtle cognitive decline compared to their premorbid level. For example, in a previous publication from our group, a small deterioration in cognitive performance has been demonstrated in patients with a single lacunar stroke (van Zandvoort et al., 2001). Although there was no generalized deterioration for the unimpaired group as a whole in the present study, a few patients developed new cognitive disorders as compared to baseline, which could be the consequence of increasing brain damage due to a growing amount of white matter lesions, cerebral atrophy, or silent infarcts (Vermeer et al., 2003). However, for the group as a whole, preexistent brain pathology was unrelated (1) to cognitive performance at baseline or (2) to the degree of cognitive recovery between baseline and follow-up.

Patients who were cognitively impaired in the early phase of stroke still performed worse than healthy controls at followup, especially if multiple cognitive disorders were present at baseline. Nevertheless, improvement as compared to baseline seemed to be the rule rather than the exception in all cognitive domains. Previous studies have reported global cognitive improvement rates ranging from $10 \%$ to $50 \%$ (Desmond et al., 1996; Tham et al., 2002; Ballard et al., 2003; Rasquin et al., 2004), depending primarily on the time interval between the stroke event and the neuropsychological assessments, and on the criteria used to define recovery. In the present study, domain-specific recovery occurred in $41 \%$ to $83 \%$ of cases, depending on the nature of the cognitive deficit. Recovery in the visual domains (i.e., in visual perception/construction and visual memory) was the most prevalent, whereas recovery in abstract reasoning and language was the least common. The prevalence of recovery in our study was much higher than in the aforementioned studies, and this is probably related to the fact that our baseline assessment occurred at a much earlier stage, leaving more room for improvement. Moreover, in contrast to previous studies (Desmond et al., 1996; Hochstenbach et al., 2003; Patel et al., 2003), we only included initially impaired patients in our estimation on the prevalence of cognitive recovery. Finally, we studied patients with a first-ever stroke without preexistent cognitive or functional problems, and this population probably has a larger potential for cognitive recovery than the stroke population in general (Jorgensen et al., 1997).

The high prevalence of domain-specific recovery in our study indicates that cognitive functions have a dynamic (adaptable) rather than a static (unchangeable) course after stroke. This finding is particularly important, as patients and their caregivers and some general practitioners often 
assume that cognitive decline cannot be restored following stroke because of the popular association with dementia. It has been argued that the mechanism underlying normal learning is the same mechanism that is implicated in the recovery of function following acquired brain damage, that is, the so-called Hebbian learning mechanism, which involves experience-dependent dendritic sprouting (Robertson \& Murre, 1999). Moreover, there are some indications of neuronal regeneration following brain damage (Eriksson et al., 1998). The location of brain plasticity responsible for cognitive recovery is still unclear however, with evidence of both peri-lesional changes as well as contralateral reorganization (Calvert et al., 2000; Perani et al., 1993), possibly also depending on the interval post-stroke and on the nature of the cognitive deficit.

Several factors emerged as important independent predictors of domain-specific cognitive recovery, with some factors associated with recovery in multiple cognitive domains and some factors associated with recovery in isolated cognitive domains. A high level of preexistent ability (NART score) was an excellent predictor of a good recovery in almost all cognitive domains. One plausible reason is that these patients are more capable of creating a suitable compensation strategy to circumvent their cognitive deficit. Moreover, it has been suggested that factors such as intelligence or education provide the brain with a "cognitive reserve capacity" (Staff et al., 2004). The concept of cognitive reserve posits that individual differences in how tasks are processed might provide a differential reserve against brain pathology or age-related changes (Stern et al., 2003). The neurobiological correlates of this "cognitive reserve" are still uncertain, but animal studies have demonstrated that exposure to a more stimulating environment is associated with a greater synaptic density and more complex neuronal connections (Kolb, 1999). According to this view, a denser connectivity implies more opportunity for cognitive recovery (e.g., Kolb, 1999; Robertson \& Murre, 1999). A younger age was also associated with a better recovery in executive functioning, language, and visual and verbal memory. According to the aforementioned view, age reduces the neuronal connectivity and might therefore result in a worse cognitive recovery after brain damage (Robertson \& Murre, 1999). Gender did not influence recovery in any of the cognitive domains in our study.

Several lesion locations were related to recovery in cognitive functioning. A lesion involving the frontal lobe was related to poorer recovery in visual perception and construction. Occipital lesions compromised recovery in visual perception and visual memory, probably related to the presence of visual field defects in these patients. Temporal lesions compromised recovery in executive functioning and verbal memory. With respect to memory, the temporal cortex is believed to be responsible for the consolidation of memory traces (Miyashita, 2004), which may explain the association we found with a poorer recovery in verbal memory. In contrast to previous studies that have reported conflicting findings with evidence of both a left and a right hemisphere advantage related to cognitive recovery (Desmond et al., 1996; Hochstenbach et al., 2003), lesion side was not a predictor of recovery in the present study. Lesion volume emerged as an independent predictor of visual memory, but not with respect to recovery in other cognitive functions. These findings indicate that lesion location affects cognitive recovery more than lesion volume when considering the simultaneous influence of multiple lesion characteristics. Prior studies have indeed shown severe cognitive impairment in patients with only small but strategically located lesions (Auchus et al., 2002; Szirmai et al., 2002).

Diabetes mellitus was related to recovery in abstract reasoning, but other vascular risk factors were not related to cognitive recovery in any domain. The link between diabetes mellitus and poor recovery in post-stroke cognitive functioning (Desmond et al., 1996) and cognitive functioning in general (Biessels et al., 2002; Kanaya et al., 2004) has been demonstrated before. Diabetes mellitus has also been identified as a risk factor for vascular dementia (Biessels et al., 2002). In this respect, it has been shown that both pathological increases and decreases of glucose concentrations may affect cognition. Also, the neuroradiological alterations in patients with diabetes mimic those observed in the aging brain, and therefore it has been suggested that diabetes causes an acceleration of the aging process in the brain (Biessels et al., 2002).

Finally, neither stroke severity, as measured with the NIHSS, nor the presence of depressive symptoms at baseline mediated cognitive recovery. In contrast, the severity of unilateral neglect immediately after stroke was related to a poor recovery in visual memory, but this relation disappeared in the multivariate model. It has been hypothesized that unilateral neglect probably arises from a combination of two deficits, that is, a rightward bias plus a deficit in spatial working memory (i.e., retaining locations already searched) (Husain et al., 2001). Our findings support this view and suggest that even when the lateral bias disappears, the visual memory capacity may still be affected in the longer term.

Some limitations of this study should be addressed. First, we only had a single follow-up examination between six and ten months after stroke. Although the largest amount of spontaneous recovery will have taken place within this time range, it is possible that we underestimated the prevalence of recovery. Alternatively, the course of cognitive functioning might change after the first six months, as one recent study has shown that $22 \%$ of patients with a stroke or TIA demonstrated a decline in cognitive functioning between 3-6 months poststroke and after one year (Sachdev et al., 2004). A potential underlying mechanism might be that the preexistent brain pathology that was initially silent continues to proceed and exacerbate cognitive performance in the longer term after stroke. Therefore, our cohort is in the process of a further follow-up to examine the nature of domain-specific cognitive change in the longer term. Second, as we were interested in associates of cognitive recovery exclusively related to the stroke, we included a selective stroke population without preexistent cognitive, neurological, or psychiatric deficits. Con- 
sequently, these findings cannot directly be generalized to the population with first-ever stroke as a whole, particularly given the fact that patients who were not included for follow-up demonstrated more cognitive impairments at baseline. In addition, 22 patients were too impaired in the early phase to be included in our study at all. This selection bias probably resulted in an underestimation of the frequency of impairment in the long term after stroke, and might have concealed predictors of cognitive recovery. In an attempt to avoid this selection bias as much as possible, we chose to administer a concise neuropsychological examination in the acute phase of stroke and to extend this examination at follow-up. It should be noted, however, that this approach might have influenced our findings with respect to the prevalence of domain-specific cognitive recovery in our sample. A final important remark is that we do not claim to have examined the degree and associates of spontaneous recovery. In this respect, it should be noted that cognitively impaired and unimpaired patients demonstrated a differential discharge destination and treatment pattern. Rather, we have examined outcome according to the status quo (standard practice) in the Netherlands, with cognitively impaired patients rightly receiving more treatment than cognitively unimpaired patients. It is sensible to assume that this probably affected outcome in cognitively impaired patients in a positive way. Moreover, cognitive rehabilitation in a certain cognitive domain (e.g., executive functioning) might be less effective than cognitive intervention with respect to another cognitive domain (e.g., language), and these differential effects might have influenced our findings to some extent. Future research in the effectiveness of cognitive rehabilitation is certainly warranted in order to disentangle treatment effects from spontaneous recovery.

In sum, identification and characterization of cognitive impairment in acute stroke provides valuable and specific prognostic information with respect to long-term cognitive functioning. The prognosis of acute higher-level visual disorders was the most favorable. Cognitive recovery after first-ever stroke was associated with younger age, higher preexistent ability, smaller lesion volume, lesion location, and diabetes mellitus.

\section{ACKNOWLEDGMENT}

This study was supported by the Netherlands Heart Foundation (2000.023).

\section{REFERENCES}

Auchus, A.P., Chen, C.P., Sodagar, S.N., Thong, M., \& Sng, E.C. (2002). Single stroke dementia: Insights from 12 cases in Singapore. Journal of the Neurological Sciences, 203-204, 85-89.

Baldo, J.V., Dronkers, N.F., Wilkins, D., Ludy, C., Raskin, P., \& Kim, J. (2005). Is problem solving dependent on language? Brain and Language, 92, 240-250.

Ballard, C., Rowan, E., Stephens, S., Kalaria, R., \& Kenny, R.A. (2003). Prospective follow-up study between 3 and 15 months after stroke: Improvements and decline in cognitive function among dementia-free stroke survivors $>75$ years of age. Stroke, 34, 2440-2444.

Biernaskie, J., Chernenko, G., \& Corbett, D. (2004). Efficacy of rehabilitative experience declines with time after focal ischemic brain injury. Journal of Neuroscience, 24, 1245-1254.

Biessels, G.J., van der Heide, L.P., Kamal, A., Bleys, R.L., \& Gispen, W.H. (2002). Ageing and diabetes: Implications for brain function. European Journal of Pharmacology, 441, 1-14.

Brott, T., Adams, H.P., Jr., Olinger, C.P., Marler, J.R., Barsan, W.G., Biller, J., Spilker, J., Holleran, R., Eberle, R., Hertzberg, V., Rorick, M., Moomaw, C.J., \& Walker, M. (1989). Measurements of acute cerebral infarction: A clinical examination scale. Stroke, 20, 864-870.

Calvert, G.A., Brammer, M.J., Morris, R.G., Williams, S.C., King, N., \& Matthews, P.M. (2000). Using fMRI to study recovery from acquired dysphasia. Brain and Language, 71, 391-399.

Clark-Carter, D. (1997). Doing quantitative psychological research. From design to report. Hove, UK: Psychology Press.

Crawford, J.R., Parker, D.M., \& Besson, J.A. (1988). Estimation of premorbid intelligence in organic conditions. British Journal of Psychiatry, 153, 178-181.

de Jonghe, J.F., Schmand, B., Ooms, M.E., \& Ribbe, M.W. (1997). Abbreviated form of the Informant Questionnaire on Cognitive Decline in the Elderly. Tijdschrift voor Gerontologie en Geriatrie, 28, 224-229.

De Renzi, E., Faglioni, P., Savoiardo, M., \& Vignolo, L.A. (1966). The influence of aphasia and of the hemispheric side of the cerebral lesion on abstract thinking. Cortex, 2, 339-420.

Desmond, D.W., Moroney, J.T., Sano, M., \& Stern, Y. (1996). Recovery of cognitive function after stroke. Stroke, 27, 1798-1803.

Elderkin-Thompson, V., Kumar, A., Mintz, J., Boone, K., Bahng, E., \& Lavretsky, H. (2004). Executive dysfunction and visuospatial ability among depressed elders in a community setting. Archives of Clinical Neuropsychology, 19, 597-611.

Eriksson, P.S., Perfilieva, E., Bjork-Eriksson, T., Alborn, A.M., Nordborg, C., Peterson, D.A., \& Gage, F.H. (1998). Neurogenesis in the adult human hippocampus. Nature Medicine , 4, 1313-1317.

Ferro, J.M. (2001). Hyperacute cognitive stroke syndromes. Journal of Neurology, 248, 841-849.

Gainotti, G., D’Erme, P., Villa, G., \& Caltagirone, C. (1986). Focal brain lesions and intelligence: A study with a new version of Raven's Colored Matrices. Journal of Clinical and Experimental Neuropsychology, 8, 37-50.

Galski, T., Bruno, R.L., Zorowitz, R., \& Walker, J. (1993). Predicting length of stay, functional outcome, and aftercare in the rehabilitation of stroke patients. The dominant role of higherorder cognition. Stroke, 24, 1794-1800.

Henon, H., Pasquier, F., Durieu, I., Godefroy, O., Lucas, C., Lebert, F., \& Leys, D. (1997). Preexisting dementia in stroke patients: Baseline frequency, associated factors, and outcome. Stroke, 28, 2429-2436.

Hillis, A.E., Barker, P.B., Wityk, R.J., Aldrich, E.M., Restrepo, L., Breese, E.L., \& Work, M. (2004). Variability in subcortical aphasia is due to variable sites of cortical hypoperfusion. Brain and Language, 89, 524-530.

Hillis, A.E., Wityk, R.J., Barker, P.B., Ulatowski, J.A., \& Jacobs, M.A. (2003). Change in perfusion in acute nondominant hemisphere stroke may be better estimated by tests of hemispatial neglect than by the National Institutes of Health Stroke Scale. Stroke, 34, 2392-2396.

Hochstenbach, J.B., den Otter, R., \& Mulder, T.W. (2003). Cogni- 
tive recovery after stroke: A 2-year follow-up. Archives of Physical Medicine and Rehabilitation, 84, 1499-1504.

Hosmer, D.W. \& Lemeshow, S. (1989). Applied logistic regression. New York: Wiley.

Husain, M., Mannan, S., Hodgson, T., Wojciulik, E., Driver, J., \& Kennard, C. (2001). Impaired spatial working memory across saccades contributes to abnormal search in parietal neglect. Brain, 124, 941-952.

Jorgensen, H.S., Nakayama, H., Reith, J., Raaschou, H.O., \& Olsen, T.S. (1997). Stroke recurrence: Predictors, severity, and prognosis. The Copenhagen Stroke Study. Neurology, 48, 891-895.

Kanaya, A.M., Barrett-Connor, E., Gildengorin, G., \& Yaffe, K. (2004). Change in cognitive function by glucose tolerance status in older adults: A 4-year prospective study of the Rancho Bernardo study cohort. Archives of Internal Medicine, 164, 1327-1333.

Kessels, R.P., Nys, G.M., Brands, A.M., \& van Zandvoort, M.J. (2004). The Location Learning Test as a measure of spatial memory: Applicability of a modified administration procedure and normative data. Tijdschrift voor Gerontologie en Geriatrie, 35, 147-152.

Kolb, B. (1999). Synaptic plasticity and the organization of behaviour after early and late brain injury. Canadian Journal of Experimental Psychology, 53, 62-76.

Laska, A.C., Hellblom, A., Murray, V., Kahan, T., \& Von Arbin, M. (2001). Aphasia in acute stroke and relation to outcome. Journal of Internal Medicine, 249, 413-422.

Lezak, M.D., Howieson, D.B., \& Loring, D.W. (2004). Neuropsychological assessment (4th ed.). New York: Oxford University Press.

Lin, J.H., Lin, R.T., Tai, C.T., Hsieh, C.L., Hsiao, S.F., \& Liu, C.K. (2003). Prediction of poststroke dementia. Neurology, 61, 343-348.

Manly, T., Woldt, K., Watson, P., \& Warburton, E. (2002). Is motor perseveration in unilateral neglect 'driven' by the presence of neglected left-sided stimuli? Neuropsychologia, 40, 1794-1803.

Miyashita, Y. (2004). Cognitive memory: Cellular and network machineries and their top-down control. Science, 306, 435-440.

Mok, V.C., Wong, A., Lam, W.W., Fan, Y.H., Tang, W.K., Kwok, T., Hui, A.C., \& Wong, K.S. (2004). Cognitive impairment and functional outcome after stroke associated with small vessel disease. Journal of Neurology, Neurosurgery, and Psychiatry, 75, 560-566.

Montgomery, S.A. \& Åsberg, M. (1979). A new depression scale designed to be sensitive to change. British Journal of Psychiatry, 134, 382-389.

Nys, G.M.S., van Zandvoort, M.J.E., de Kort, P.L.M., van der Worp, H.B., Jansen, B.P.W., Algra, A., de Haan, E.H.F., \& Kappelle, L.J. (2005). The prognostic value of domain-specific cognitive abilities in acute first-ever stroke. Neurology, 64, 821-827.

Paolucci, S., Antonucci, G., Grasso, M.G., Morelli, D., Troisi, E., Coiro, P., \& Bragoni, M. (2000). Early versus delayed inpatient stroke rehabilitation: A matched comparison conducted in Italy. Archives of Physical Medicine and Rehabilitation, 81, 695-700.

Patel, M., Coshall, C., Rudd, A.G., \& Wolfe, C.D. (2003). Natural history of cognitive impairment after stroke and factors associated with its recovery. Clinical Rehabilitation, 17, 158-166.

Pedersen, P.M., Jorgensen, H.S., Nakayama, H., Raaschou, H.O., $\&$ Olsen, T.S. (1995). Aphasia in acute stroke: Incidence, determinants, and recovery. Annals of Neurology, 38, 659-666.
Perani, D., Vallar, G., Paulesu, E., Alberoni, M., \& Fazio, F. (1993). Left and right hemisphere contribution to recovery from neglect after right hemisphere damage:An [18F]FDG PET study of two cases. Neuropsychologia, 31, 115-125.

Rasquin, S.M., Lodder, J., Ponds, R.W., Winkens, I., Jolles, J., \& Verhey, F.R. (2004). Cognitive functioning after stroke: A oneyear follow-up study. Dementia and Geriatric Cognitive Disorders, 18, 138-144.

Robertson, I.H. \& Murre, J.M. (1999). Rehabilitation of brain damage: Brain plasticity and principles of guided recovery. Psychological Bulletin, 125, 544-575.

Robertson, I.H., Ward, T., Ridgeway, V., \& Nimmo-Smith, I. (1994). The Test of Everyday Attention. Bury St. Edmunds, UK: Thames Valley Test Company.

Rusconi, M.L., Maravita, A., Bottini, G., \& Vallar, G. (2002). Is the intact side really intact? Perseverative responses in patients with unilateral neglect: A productive manifestation. Neuropsychologia, 40, 594-604.

Sachdev, P.S., Brodaty, H., Valenzuela, M.J., Lorentz, L.M., \& Koschera, A. (2004). Progression of cognitive impairment in stroke patients. Neurology, 63, 1618-1623.

Schmand, B., Bakker, D., Saan, R., \& Louman, J. (1991). The Dutch Reading Test for Adults: A measure of premorbid intelligence level. Tijdschrift voor Gerontologie en Geriatrie, 22, 15-19.

Staff, R.T., Murray, A.D., Deary, I.J., \& Whalley, L.J. (2004). What provides cerebral reserve? Brain, 127, 1191-1199.

Stern, Y., Zarahn, E., Hilton, H.J., Flynn, J., DeLaPaz, R., \& Rakitin, B. (2003). Exploring the neural basis of cognitive reserve. Journal of Clinical and Experimental Neuropsychology, 25, 691-701.

Szirmai, I., Vastagh, I., Szombathelyi, E., \& Kamondi, A. (2002). Strategic infarcts of the thalamus in vascular dementia. Journal of the Neurological Sciences, 203-204, 91-97.

Tatemichi, T.K., Desmond, D.W., Stern, Y., Paik, M., Sano, M., \& Bagiella, E. (1994). Cognitive impairment after stroke: Frequency, patterns, and relationship to functional abilities. Journal of Neurology, Neurosurgery, and Psychiatry, 57, 202-207.

Tham, W., Auchus, A.P., Thong, M., Goh, M.L., Chang, H.M., Wong, M.C., \& Chen, C.P. (2002). Progression of cognitive impairment after stroke: One year results from a longitudinal study of Singaporean stroke patients. Journal of the Neurological Sciences, 203-204, 49-52.

van der Worp, H.B., Claus, S.P., Bar, P.R., Ramos, L.M., Algra, A., van Gijn, J., \& Kappelle, L.J. (2001). Reproducibility of measurements of cerebral infarct volume on CT scans. Stroke, 32, 424-430.

van Swieten, J.C., Koudstaal, P.J., Visser, M.C., Schouten, H.J., \& van Gijn, J. (1988). Interobserver agreement for the assessment of handicap in stroke patients. Stroke, 19, 604-607.

van Zandvoort, M.J.E., de Haan, E.H.F., \& Kappelle, L.J. (2001). Chronic cognitive disturbances after a single supratentorial lacunar infarct. Neuropsychiatry, Neuropsychology, and Behavioral Neurology, 14, 98-102.

Vermeer, S.E., Prins, N.D., den Heijer, T., Hofman, A., Koudstaal, P.J., \& Breteler, M.M. (2003). Silent brain infarcts and the risk of dementia and cognitive decline. New England Journal of Medicine, 348, 1215-1222.

Watt, K.J., \& O'Carroll, R.E. (1999). Evaluating methods for estimating premorbid intellectual ability in closed head injury. Journal of Neurology, Neurosurgery, and Psychiatry, 66, 474-479.

Wilson, B., Cockburn, J., \& Halligan, P. (1987). Development of a behavioral test of visuospatial neglect. Archives of Physical Medicine and Rehabilation, 68, 98-102. 